\title{
Discovery of an X-Ray Quasar Wind Driving the Cold Gas Outflow in the Ultraluminous Infrared Galaxy IRAS F05189-2524
}

\author{
Robyn N. Smith ${ }^{1}$ (10), Francesco Tombesi ${ }^{1,2,3,4}$ (1) , Sylvain Veilleux ${ }^{1,5,6}$ (1) , Anne M. Lohfink ${ }^{7}$, and Alfredo Luminari ${ }^{2,4}$ \\ ${ }^{1}$ Department of Astronomy, University of Maryland, College Park, MD 20742, USA; rnsmith@astro.umd.edu \\ ${ }^{2}$ Department of Physics, University of Rome "Tor Vergata," Via della Ricerca Scientifica 1, I-00133 Rome, Italy \\ ${ }^{3}$ NASA/Goddard Space Flight Center, Code 662, Greenbelt, MD 20771, USA \\ ${ }^{4}$ INAF Osservatorio Astronomico di Roma, Via Frascati 33, I-00078 Monteporzio Catone, Italy \\ 5 Joint Space-Science Institute, University of Maryland, College Park, MD 20742, USA \\ ${ }^{6}$ Institute of Astronomy and Kavli Institute for Cosmology Cambridge, University of Cambridge, Cambridge, CB3 0HA, UK \\ ${ }^{7}$ Montana State University, Department of Physics, P.O. Box 173840, Bozeman, MT 59717, USA \\ Received 2019 February 20; revised 2019 October 14; accepted 2019 October 16; published 2019 December 11
}

\begin{abstract}
We present new XMM-Newton and NuSTAR observations of the galaxy merger IRAS F05189-2524, which is classified as an ultraluminous infrared galaxy and optical Seyfert 2 at $z=0.0426$. We test a variety of spectral models that yield a best fit consisting of an absorbed power law with emission and absorption features in the Fe K band. Remarkably, we find evidence for a blueshifted Fe K absorption feature at $E=7.8 \mathrm{keV}$ (rest frame) which implies an ultrafast outflow (UFO) with $v_{\text {out }}=0.11 \pm 0.01 c$. We calculate that the UFO in IRAS F05189-2524 has a mass outflow rate of $\dot{M}_{\text {out }} \gtrsim 1.0 M_{\odot} \mathrm{yr}^{-1}$, a kinetic power of $\dot{E}_{\mathrm{K}} \gtrsim 8 \% L_{\mathrm{AGN}}$, and a momentum rate (or force) of $\dot{P}_{\text {out }} \gtrsim 1.4 L_{\mathrm{AGN}} / c$. Comparing the energetics of the UFO to the observed multi-phase outflows at kiloparsec scales yields an efficiency factor of $f \sim 0.05$ for an energy-driven outflow. Given the uncertainties, however, we cannot exclude the possibility of a momentum-driven outflow. Comparing IRAS F05189-2524 with nine other objects with observed UFOs and large-scale galactic outflows suggests that there is a range of efficiency factors for the coupling of the energetics of the nuclear and galaxy-scale outflows that likely depend on specific physical conditions in each object.
\end{abstract}

Key words: galaxies: active - galaxies: individual (IRAS F05189-2524) - quasars: absorption lines - X-rays: galaxies

\section{Introduction}

In one possible evolutionary scenario, gas-rich galaxies merge together to form an obscured ultraluminous infrared galaxy (ULIRG), which evolves into a dusty quasar and then eventually an exposed optical quasar after shedding its gas and dust cocoon (e.g., Sanders et al. 1988; Veilleux et al. 2002, 2009a, 2009b; Hopkins et al. 2006). This scenario may account for the intimate link between the mass of the stellar spheroid component of the host galaxy and that of the central supermassive black hole (SMBH; e.g., Silk \& Rees 1998; Ferrarese \& Merritt 2000; Gebhardt et al. 2000) by invoking negative feedback of the active galactic nucleus (AGN) i.e., the AGN drives galactic winds that in turn may be able to quench the growth of both the SMBH and the stellar component of the host (e.g., Fabian 1999; King 2003; King \& Pounds 2003).

Star formation is inhibited if the cold molecular gas out of which stars form is affected by such outflows. Far-infrared molecular spectroscopy of ULIRGs has revealed highly blueshifted absorption features indicative of high-velocity molecular outflows on scales of hundreds of parsecs, which implies significant mass outflow rates (Sturm et al. 2011; Veilleux et al. 2013, 2017; González-Alfonso et al. 2017; Rupke et al. 2017). Most models explaining the origin of these galactic outflows require a very fast $\left(v_{\text {out }} \sim 0.1 c\right)$ initial AGN accretion disk wind that shocks the surrounding interstellar medium (ISM) and forms a hot bubble that moves the molecular material (see King \& Pounds 2015 and references therein). The shock-driven galactic outflow can be divided into two distinct regimes: momentum-driven and energy-driven.
Momentum-driven outflows occur when the kinetic energy of the wind is mostly radiated away, in which case, only ram pressure exerts work on the surrounding ISM. Energy-driven outflows occur if the shocked ISM is not efficiently cooled and expands adiabatically as a hot bubble. The momentum rate of an energy-driven outflow is expected to be larger than that of a momentum-driven outflow and may approach values of $\dot{P} \simeq 10 L_{\mathrm{AGN}} / c$, which is consistent with observations of several ULIRGs (Sturm et al. 2011; Cicone et al. 2014; González-Alfonso et al. 2017).

Galactic-scale outflows are common in (U)LIRGs and often involve several gas phases: the molecular gas (e.g., Veilleux et al. 2013; González-Alfonso et al. 2017; Fluetsch et al. 2019), the neutral atomic gas (Rupke \& Veilleux 2013; Teng et al. 2013; Rupke et al. 2017), the warm ionized gas (Rupke \& Veilleux 2013; Rupke et al. 2017), and sometimes even the hot ionized gas (Nardini et al. 2013; Veilleux et al. 2014; Paggi et al. 2017; Liu et al. 2019). Conversely, outflows inferred from blueshifted Fe XXV / XXVI absorption lines in the X-ray band at rest-frame energies $E>7 \mathrm{keV}$ are observed in AGNs at subparsec scales consistent with an accretion disk interpretation. These ultrafast outflows (UFOs; Tombesi et al. 2010, 2011, 2015; Gofford et al. 2013; Tombesi \& Cappi 2014; Longinotti et al. 2015; Nardini et al. 2015; Parker et al. 2017) have outflow velocities that are mildly relativistic $\left(v_{\text {out }} \sim 0.1 c\right)$. Confirming both a large-scale galactic outflow and sub-parsec scale accretion disk wind in the same object presents observational challenges requiring simultaneous detection of the outflow in the X-rays and at lower energies (mm-optical-IR). 
IRAS F11119+3257 was the first such source in which both outflows were confirmed. Galactic outflows were found using $\mathrm{OH}$ absorption measurements with Herschel (Veilleux et al. 2013) and confirmed with $\mathrm{CO}(1-0)$ emission line measurements from deep ALMA observations (Veilleux et al. 2017). The UFO was initially detected with Suzaku (Tombesi et al. 2015) and later confirmed with NuSTAR observations (Tombesi et al. 2017). Mrk 231 is the second known object whose outflows were confirmed using IRAM, Chandra, and NuSTAR (Feruglio et al. 2015). Therefore, it is imperative to extend such studies to other sources in order to quantify the occurrence of such phenomena.

\section{IRAS F05189-2524}

IRAS F05189-2524 is a well-studied, nearby $(z=0.0426)$, late-stage merger ULIRG (Veilleux et al. 2002, 2006). It is an optical Seyfert 2 (Veilleux et al. 1999a), but contains hidden broadline $\mathrm{Pa} \beta$ in the near-infrared (Veilleux et al. 1999b). With $\sim 70 \%$ of its bolometric luminosity $\left(L_{\text {bol }} \sim 10^{12} L_{\odot}\right)$ attributed to its AGN (Veilleux et al. 2009b), the AGN in IRAS F051892524 is considered a quasar. A high-velocity, large-scale outflow has been detected in the neutral, ionized, and molecular gas phases (Rupke et al. 2005, 2017; Westmoquette et al. 2012; Bellocchi et al. 2013; Teng et al. 2013; Veilleux et al. 2013; González-Alfonso et al. 2017).

In the X-ray, IRAS F05189-2524 is one of the brightest local ULIRGs. Archival XMM-Newton and Chandra observations derive an $E=2-10 \mathrm{keV}$ continuum luminosity of $\sim 10^{43} \mathrm{erg}$ $\mathrm{s}^{-1}$ (Teng et al. 2009). The X-ray flux of IRAS F05189-2524 is known to vary. The $E=0.5-2 \mathrm{keV}$ flux was relatively constant during XMM-Newton observations in 2001 March, Chandra observations in 2001 October and 2002 January, and Suzaku observations in 2006 April. The $E=2-10 \mathrm{keV} \mathrm{flux}$, however, was a factor of $\sim 30$ lower in the 2006 Suzaku than previously measured in the XMM-Newton and Chandra observations in 2001-02. In addition to the drop in flux, the 2006 Suzaku observation revealed a prominent $E=6.4 \mathrm{keV} \mathrm{Fe} \mathrm{K} \mathrm{emission}$ line not seen in the 2001-02 observations (Teng et al. 2009). Observations by ASCA in 1995 and BeppoSAX in 1999 found statistically significant unresolved iron line emission, but also confirmed strong continuum variability above $E=2 \mathrm{keV}$ between the two observations (Severgnini et al. 2001). IRAS F05189-2524 was observed by NuSTAR in 2013 February (21 $\mathrm{ks})$ and October (25 and $8 \mathrm{ks}$ ) with a coordinated XMM-Newton observation during the 2013 October observation $(31 \mathrm{ks}$; Teng et al. 2015). Minor flux variations detected between these observations were not found to be statistically significant, and the $E=2-10 \mathrm{keV}$ flux was again consistent with the "high" state of the 2001-02 observations (Teng et al. 2015).

IRAS F05189-2524 was detected by Swift BAT with a significance of $6 \sigma$ at $E=14-195 \mathrm{keV}$ and $4.2 \sigma$ at $E=$ 24-35 keV (Koss et al. 2013). In reanalyzing the $2013 \mathrm{NuSTAR}$ and XMM-Newton observations, $\mathrm{Xu}$ et al. (2017) found that IRAS F05189-2524 may be modeled above $E=2 \mathrm{keV}$ by a broad iron line disk reflection. Xu et al. (2017) also found that possible features indicative of a high-velocity outflow in the $\mathrm{Fe}$ $\mathrm{K}$ band are not statistically required after the fit with a relativistic reflection dominated spectral model. Data of higher quality are needed to confirm the possible existence of these spectral features.
Table 1

Exposure Times and Count Rates for XMM-Newton Observation (ID 0790580101) and NuSTAR Observation (ID 60201022002) of IRAS F05189-2524.

\begin{tabular}{lccc}
\hline \hline Instrument & $\begin{array}{c}\text { Exposure } \\
(\mathrm{ks})\end{array}$ & $\begin{array}{c}\text { Count Rate } \\
0.5-2 \mathrm{keV}\end{array}$ & $\begin{array}{c}\text { Count Rate } \\
2-10 \mathrm{keV}^{\mathrm{a}}\end{array}$ \\
\hline EPIC pn & 74.33 & 0.393 & 0.315 \\
EPIC MOS & 94.71 & 0.251 & 0.212 \\
FPMA (full) & 144.1 & & 0.067 \\
FPMB (full) & 143.9 & & 0.061 \\
FPMA (simultaneous) & 45.39 & & 0.073 \\
FPMB (simultaneous) & 45.31 & 0.066 \\
\hline
\end{tabular}

Note.

${ }^{\text {a }}$ Count rates for NUSTAR are calculated between 3 and $10 \mathrm{keV}$.

\section{Observations and Data Reduction}

\subsection{XMM-Newton}

IRAS F05189-2524 was observed by XMM-Newton for $98 \mathrm{ks}$ on 2016 September 6-7 (ObsID 0790580101) with the EPIC pn and MOS cameras. The observations were reduced using standard procedures with the XMM-Newton Science Analysis System v16.1.0. Soft proton flares were removed, and only single and double events were retained for the pn while single through quadruple events were retained for the MOS. The source was extracted using a $40^{\prime \prime}$ radius circular region. The background was estimated from a source-free sky region of the same size. For the pn background, special care was taken to ensure that the background region was not located on parts of the charged coupled device (CCD) where there are known instrumental X-ray fluorescent lines (Freyberg et al. 2004), particularly the $\mathrm{Cu}-\mathrm{K} \alpha$ line around $8 \mathrm{keV}$. The final good exposure time for the pn was $74.3 \mathrm{ks}$. The MOS1 and MOS2 observations were reduced separately. Each MOS spectrum and light curve was inspected individually, and finding no gross variability between the two, they were combined using epicspeccombine. The final good exposure time for the combined MOS spectrum is $94.7 \mathrm{ks}$. Table 1 provides the final good exposure times and count rates for the XMM-Newton observation. The final spectrum for both the pn and MOS were grouped to a minimum of 50 counts per bin in order to ensure the use of the $\chi^{2}$ statistics.

\subsection{NUSTAR}

IRAS F05189-2524 was observed by NuSTAR for $144 \mathrm{ks}$ on 2016 September 5-8 (ObsID 60201022002). Spectra were created using HEAsoft version 6.22 and CALDB version "20171002" after initially producing cleaned event files with the tool nupipeline. For the screening parameters, we assumed "saacalc $=2$ saamode $=$ optimized tentacle $=$ yes" based on the NuSTAR SAA filtering report. From the cleaned event files, spectra and corresponding response matrices were then created using the nuproducts tool. The source region was chosen to be circular with a $60^{\prime \prime}$ radius, the background region was also circular with a $121^{\prime \prime}$ radius. The resulting spectra have a net exposure of $144.1 \mathrm{ks}$ for focal plane module FPMA (focal plane module A) and $143.9 \mathrm{ks}$ for FPMB. Due to differing orbits, the NuSTAR observation is only strictly concurrent with $X M M$-Newton for $45 \mathrm{ks}$. Table 1 provides the final good exposure times and count rates for the NUSTAR observation. All FPMA and FPMB spectra were grouped to a minimum of 25 counts per bin in order to ensure the use of the $\chi^{2}$ statistics. 


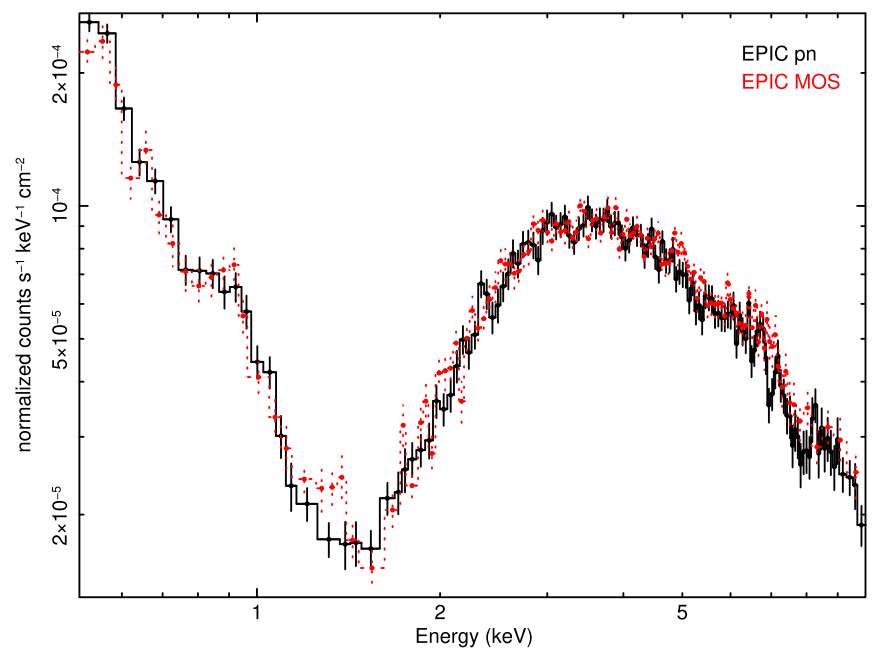

Figure 1. XMM-Newton EPIC spectrum of IRAS F05189-2524 from 0.5 to $10 \mathrm{keV}$ in the rest frame $(z=0.0426)$. Additional binning has been applied for visual purposes.

\section{XMM-Newton Spectral Analysis}

We perform our spectral analysis using XSPEC v12.10 c (Arnaud 1996) using $\chi^{2}$ statistics. All models take into account Galactic absorption with the tbabs model (Wilms et al. 2000) using a Galactic column density of $N_{\mathrm{H}, \mathrm{Gal}}=1.66 \times 10^{20} \mathrm{~cm}^{-2}$ (Kalberla et al. 2005). All parameters are given in the rest frame of IRAS F05189-2524 $(z=0.0426)$. The full XMMNewton EPIC spectrum of IRAS F05189-2524 from 0.5$10 \mathrm{keV}$ is presented in Figure 1. All errors and limits are given at a level of $90 \%\left(\Delta \chi^{2}=2.7\right.$ for one degree of freedom). Statistical calculations were performed using XSPEC error and steppar commands avoiding local minima when searching $\chi^{2}$ space. The difference in sensitivity of the pn and MOS spectra are due to the difference in effective area. The effective area of the MOS decreases more rapidly at higher energies than the pn.

\subsection{Broadband Modeling}

We begin by joint modeling the EPIC pn and MOS spectra from 0.5 to $10 \mathrm{keV}$ with a simple power law. This provides a poor fit $\left(\chi_{\text {red }}^{2}=\chi^{2} / \nu=11.93\right)$ and is not considered further. The spectrum is indicative of a soft X-ray absorber (see Figure 1), so our next model invokes a full covering neutral absorber (zwabs in XSPEC). While this provides a better fit $\left(\chi_{\text {red }}^{2}=6.47\right)$, it is clear that the model is not accounting for any emission that is present at soft X-ray energies $(E<2 \mathrm{keV})$ and is not considered further.

We then consider a neutral partial covering absorber (zpcfabs), which provides a significant improvement in the overall fit $\left(\chi_{\text {red }}^{2}=1501 / 948=1.58\right.$; see Figure $\left.2(a)\right)$ although there is still excess emission at soft energies. This model has a column density $N_{\mathrm{H}}=(8.54 \pm 0.12) \times 10^{22} \mathrm{~cm}^{-2}$, and the photon index, while high $(\Gamma=2.29 \pm 0.01)$, is not unreasonable given the large range of previously published values for IRAS F05189-2524 (Risaliti et al. 2000; Ptak et al. 2003; Teng et al. 2015; Xu et al. 2017). We also test a continuum scattering model using two power laws with the same photon index, one with full neutral absorption and one with no absorption. The fit of this model is comparable to that with neutral partial covering absorption with no clear preference for either model.
Although these models are phenomenologically distinct, they are mathematically equivalent, and we will continue with our spectral analysis using neutral partial covering absorption.

As IRAS F05189-2524 is a ULIRG, we add a mekal component to account for the hot diffuse gas likely present in the host galaxy (see Figure 2(b)). The mekal component has a plasma temperature of $k T=0.181 \pm 0.004 \mathrm{keV}$ while the neutral partial covering absorber has a column density of $N_{\mathrm{H}}=(7.29 \pm 0.10) \times 10^{22} \mathrm{~cm}^{-2}$. This improves the fit to $\chi_{\text {red }}^{2}=1050 / 946=1.11$ in addition to yielding a photon index of $\Gamma=1.97 \pm 0.01$, much closer to the canonical value of $\Gamma=2$ (e.g., Nandra \& Pounds 1994; Reeves \& Turner 2000). Both neutral partial covering absorber models (with and without the mekal component) have a covering fraction of $98 \%$ with full covering excluded at the $90 \%$ level.

Next, we test an ionized partial covering absorber (zxipcf), which slightly improves the fit $\left(\chi_{\text {red }}^{2}=1034 / 945=1.09\right.$; see Figure 2(c)). The column density increases to $N_{\mathrm{H}}=$ $(11.06 \pm 1.10) \times 10^{22} \mathrm{~cm}^{-2}$ with a low ionization parameter of $\log \xi=0.59 \pm 0.05 \mathrm{erg} \mathrm{s}^{-1} \mathrm{~cm}$ and a covering fraction of $99 \%$ (full covering remains excluded at the $90 \%$ level). The plasma temperature of the mekal component decreases slightly to $k T=0.147_{-0.024}^{+0.016} \mathrm{keV}$. Now, however, the continuum above $E=8 \mathrm{keV}$ is noticeably underestimated (see Figure 2(c)) while the photon index has steepened $(\Gamma=2.49 \pm 0.12)$. This is consistent with the ionized partial covering absorber compromising the continuum fit for the sake of the large contribution to the residuals at lower energies. For these reasons, we discard the model with the ionized partial covering absorber.

From the data-to-model ratios in Figure 2, we find evidence for a possible absorption feature between $E \sim 7-8 \mathrm{keV}$. We approximate this feature by adding a Gaussian to our model with a neutral partial covering absorber and mekal component (see Figure 2(d)). The center of the line is located at $E=7.81 \pm 0.10 \mathrm{keV}$ with a width of $\sigma=103 \mathrm{eV}(90 \%$ upper limit $\sigma<248 \mathrm{eV}$ ) and an equivalent width of $56_{-34}^{+37} \mathrm{eV}$. This improves the fit by $\Delta \chi^{2} / \Delta \nu=9 / 3$, which corresponds to a statistical requirement of $97 \%$ according to the F-test ( $>$ $2 \sigma)$. The column density is $N_{\mathrm{H}}=(7.22 \pm 0.10) \times 10^{22} \mathrm{~cm}^{-2}$, covering fraction is $98 \%$, photon index $\Gamma=1.94 \pm 0.01$, and plasma temperature $k T=0.181 \pm 0.004 \mathrm{keV}$.

In our last broadband model, we add an unresolved Gaussian emission line at $E=6.70 \pm 0.06 \mathrm{keV}$ with a width frozen to $\sigma=10 \mathrm{eV}$, consistent with iron-K lines of highly ionized iron (Fe XVIII and above; Kallman et al. 2004; see Figure 2(e)). The addition of the emission line narrows the absorption feature to $\sigma=78 \mathrm{eV}$ (90\% upper limit $\sigma<240 \mathrm{eV}$ ). The equivalent width of the absorption feature also decreases to $46_{-39}^{+36} \mathrm{eV}$, while the equivalent width of the emission feature is $35 \pm 17 \mathrm{eV}$. Using an F-test, the addition of a second Gaussian is statistically significant at a level of $99.7 \%(\sim 3 \sigma)$. Other model parameters remain largely unchanged by the inclusion of an emission feature. The parameters of this best-fitting broadband model $\left(\chi_{\text {red }}^{2}=1028 / 941=1.09\right)$ are presented in Table 2.

\subsection{Modeling the Iron-K Region}

To more closely model the iron-K region, we consider the pn and MOS only between $E=2-10 \mathrm{keV}$, consistent with the methods presented in $\mathrm{Xu}$ et al. (2017) and Braito et al. (2018). From Figure 2, it is clear that the residuals are dominated by a complex array of features below $E=2 \mathrm{keV}$, some of which 


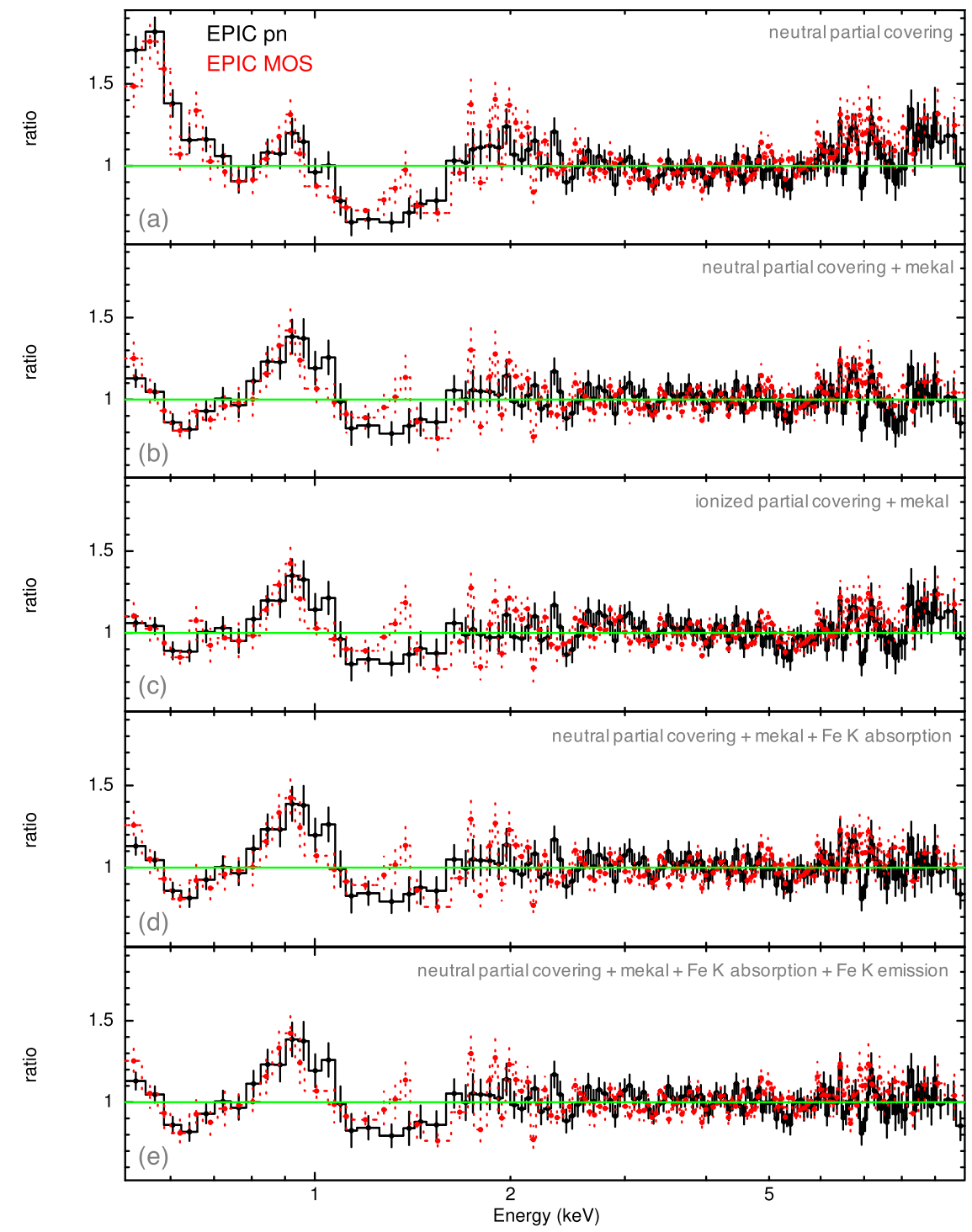

Figure 2. Data-to-model ratios for broadband models; EPIC pn is plotted in black, the MOS in red. (a) a neutral partial covering absorber by itself does not account for excess emission below $E=1 \mathrm{keV}$ and underestimates the continuum above $E=8 \mathrm{keV}$; (b) adding a mekal component to account for hot diffuse gas improves issues seen in (a); (c) testing an ionized neutral absorber results in an underestimate of the continuum above $E=8 \mathrm{keV}$; (d) a Gaussian is added to (b) to model an absorption feature at $E=7.8 \mathrm{keV}$; (e) a Gaussian is added to (d) to model an emission feature at $E=6.7 \mathrm{keV}$.

may be due to a photoionized emitter. Modeling the soft X-ray emission does not impact results from the hard X-ray emission, although the inclusion of the softer energies may detrimentally influence the continuum estimation. Investigating the source of the soft X-ray emission is not our primary objective and is not considered further in this paper.

We begin modeling the iron- $\mathrm{K}$ region with a powerlaw continuum and a neutral partial covering absorber (see Figure 3(a)). We do not include the mekal component, as it does not contribute above $E=2 \mathrm{keV}$. The column density is $N_{\mathrm{H}}=\left(7.28_{-0.27}^{+0.12}\right) \times 10^{22} \mathrm{~cm}^{-2}$ and the photon index $\Gamma=$ $1.94 \pm 0.02$. The covering fraction is $0.98 \pm 0.01$.

Next, we add a Gaussian absorption feature at $E=7.81 \pm$ $0.12 \mathrm{keV}$ with a width of $\sigma=143_{-98}^{+132} \mathrm{eV}$ and equivalent width of $72_{-38}^{+42} \mathrm{eV}$ (see Figure 3(b)). This improves the fit of the model $\left(\Delta \chi^{2} / \Delta \nu=13 / 3\right)$, and using an F-test, the addition is statistically significant at a level of $99.7 \%(\sim 3 \sigma)$. The column density is $N_{\mathrm{H}}=(6.96 \pm 0.12) \times 10^{22} \mathrm{~cm}^{-2}$ and the photon index $\Gamma=1.88 \pm 0.02$. The covering fraction is $0.984{ }_{-0.011}^{+0.014}$ (full covering remains excluded at the $90 \%$ level).

Finally, we add a second Gaussian emission feature at $E=6.70 \pm 0.06 \mathrm{keV}$ with a fixed width of $\sigma=10 \mathrm{eV}$ (see Figure 3(c)). This improves the fit of the model $\left(\Delta \chi^{2} / \Delta \nu=9 / 2\right)$, and using an F-test, the addition is statistically significant at a level of $99.3 \%$. The addition of a second Gaussian narrows the first Gaussian to $\sigma=117 \mathrm{eV}$ ( $90 \%$ upper limit $\sigma<257 \mathrm{eV}$ ) and equivalent width of $61_{-38}^{+40} \mathrm{eV}$. The column density increases slightly to $N_{\mathrm{H}}=\left(7.03_{-0.29}^{+0.12}\right) \times$ $10^{22} \mathrm{~cm}^{-2}$ and the photon index steepens slightly to $\Gamma=1.91 \pm$ 0.02 . The covering fraction remains at $0.984{ }_{-0.011}^{+0.014}$ with full covering excluded at the $90 \%$ level. The final parameters for this best-fit model are provided in Table 3 .

In order to better assess the significance of the detection of the Gaussian absorption feature at $E=7.81 \mathrm{keV}$, we run a series of detailed Monte Carlo simulations, according to the procedure described in Tombesi et al. (2010), quantifying the 
Table 2

Parameters for The Best-fitting Broadband Model

\begin{tabular}{|c|c|c|c|c|c|}
\hline Component & Parameter & Unit & Model & $\chi^{2} / \nu$ & $\Delta \chi^{2} / \Delta \nu$ \\
\hline zpowerlw & $\begin{array}{c}\Gamma \\
z^{\mathrm{a}}\end{array}$ & & $\begin{array}{c}1.97 \pm 0.01 \\
0.0426\end{array}$ & $11334 / 950$ & $\ldots$ \\
\hline zpcfabs $^{\text {b }}$ & $\begin{array}{c}N_{\mathrm{H}} \\
\text { Covering Fraction } \\
z^{\mathrm{a}}\end{array}$ & $10^{22} \mathrm{~cm}^{-2}$ & $\begin{array}{c}7.26 \pm 0.10 \\
0.984 \pm 0.001 \\
0.0426\end{array}$ & $1933 / 948$ & $9401 / 2$ \\
\hline mekal & $k T$ & $\mathrm{keV}$ & $0.181 \pm 0.004$ & $1050 / 946$ & $883 / 2$ \\
\hline zgauss & $\begin{array}{c}\text { Line } E \\
\sigma \\
z^{\mathrm{a}} \\
\text { EW }\end{array}$ & $\begin{array}{l}\mathrm{keV} \\
\mathrm{keV} \\
\mathrm{eV}\end{array}$ & $\begin{array}{c}7.81 \pm 0.06 \\
<0.24 \\
0.0426 \\
-46_{-36}^{+29}\end{array}$ & $1041 / 943$ & $9 / 3$ \\
\hline zgauss & $\begin{array}{c}\text { Line } E \\
\sigma^{\mathrm{a}} \\
z^{\mathrm{a}} \\
\mathrm{EW}\end{array}$ & $\begin{array}{l}\mathrm{keV} \\
\mathrm{keV} \\
\mathrm{eV}\end{array}$ & $\begin{array}{c}6.70 \pm 0.06 \\
0.01 \\
0.0426 \\
35 \pm 17\end{array}$ & $1028 / 941$ & $13 / 2$ \\
\hline
\end{tabular}

Notes. All errors are given at the $90 \%$ level while limits are given at $90 \%$.

a Parameters frozen at their stated values.

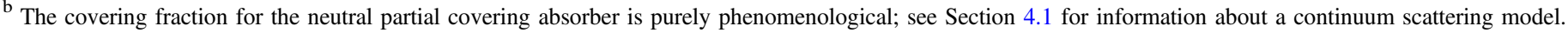

incidence of spurious lines when blindly searching for features between $E=7-10 \mathrm{keV}$ (rest frame). We adopt the best-fit model shown in Table 3 after removing the Gaussian absorption lines as our baseline model. We simulate a set of 1000 observations with both the EPIC pn and MOS detectors using the same observation times as given in Table 1 and grouping the spectra to a minimum of 50 counts per bin.

First, we fit the simulated data using our baseline model checking that the best-fit values agree within the uncertainties with the input parameters used to generate the data. Then, we look for the probability of detecting an emission or absorption Gaussian feature between $E=7-10 \mathrm{keV}$ due to random fluctuations of the simulated data. To do this, we add a Gaussian component to Model A with a line centroid restricted to fall between $E=7-10 \mathrm{keV}$ in steps of $0.1 \mathrm{keV}$. The width of the Gaussian line is free to vary between $\sigma=0-300 \mathrm{eV}$. The line normalization is left free to vary during the fit between positive and negative values, thus allowing for the presence of emission or absorption features, respectively.

Using the value of $\Delta \chi^{2}=13$ as the threshold value, we find that 8 out of $1000(f=0.008)$ simulated spectra include spurious lines which improve the fit by a greater or equal amount. We derive the confidence level of the observed absorption line as $p=1-f=0.992$, corresponding to $99.2 \%$ or $2.5 \sigma$.

The significant presence of an absorption feature above $E=7 \mathrm{keV}$ could be indicative of a UFO. The strongest highly ionized iron transitions are $\mathrm{Fe} \mathrm{XXV} \mathrm{He} \alpha(E=6.697 \mathrm{keV})$ and $\mathrm{He} \beta(E=7.880 \mathrm{keV})$ and Fe XXVI Ly $\alpha(E=6.966 \mathrm{keV})$ and $\operatorname{Ly} \beta(E=8.250 \mathrm{keV})$. For an absorption feature at $E=$ $7.8 \mathrm{keV}$ (rest frame), only Fe XXV He $\alpha$ and Fe XXVI Ly $\alpha$ would produce an outflow with velocities of $v_{\text {out }}=0.15 c$ and $v_{\text {out }}=0.11 c$, respectively.

We note an apparent narrow absorption feature at $E=$ $7 \mathrm{keV}$. However, the EPIC pn and MOS data are not consistent at that energy, and any attempt to fit a Gaussian absorption feature is consistent with a width of $\sigma=0 \mathrm{eV}$. We conclude that this faint absorption feature may be due to random fluctuations. We also note apparent narrow emission features at $E=6.4$ and $7.2 \mathrm{keV}$. These also are not statistically significant with the current data, but they are close to the expected energies for $\mathrm{Fe} \mathrm{K} \alpha$ and $\mathrm{Fe} \mathrm{K} \beta$. They will not be considered further here.

\subsection{Detailed Photoionization Modeling of the Fe K Absorber}

We perform a self-consistent photoionization modeling of the Fe $\mathrm{K}$ absorber using absorption tables generated with the photoionization code XSTAR (Kallman \& Bautista 2001) with standard solar abundances (Asplund et al. 2009). The output parameters of the XSTAR fit are the column density, ionization parameter, and the observed absorber redshift $z_{o}$. The ionization parameter is defined as $\xi=L_{\text {ion }} /\left(n r^{2}\right) \mathrm{erg} \mathrm{s}^{-1} \mathrm{~cm}$ (Tarter et al. 1969), where $L_{\text {ion }}$ is the ionizing luminosity from 1-1000 $\mathrm{Ry}^{8}$ and and $r, n$ are the distance from the central source and the number density of the gas, respectively. The observed absorber redshift is related to the intrinsic absorber redshift in the source rest frame $z_{a}$ as $\left(1+z_{o}\right)=\left(1+z_{a}\right)(1+$ $z_{c}$ ), where $z_{c}$ is the cosmological redshift of the source. The velocity can then be determined using the relativistic Doppler formula, $1+z_{a}=[(1-\beta) /(1+\beta)]^{1 / 2}$, where $\beta=v / c$.

In order to best fit the observed width of the absorption feature, we consider three absorption tables with turbulent broadening velocities of 1000,5000 , and $10,000 \mathrm{~km} \mathrm{~s}^{-1}$. All fits include the neutral partial covering absorber and a Gaussian emission line $E=6.7 \mathrm{keV}$. The XSTAR absorber well describes the observed absorption feature at $E=7.8 \mathrm{keV}$ without the need for additional Gaussian components.

Our best-fit model has a $v_{\text {turb }}=5000 \mathrm{~km} \mathrm{~s}^{-1}$. Model parameters are given in Table 4 . Figure 4 shows the datato-model ratios of models with and without the XSTAR component. The redshift of the absorber is well constrained at $z_{o}=-0.071 \pm 0.012$ (see Figure 5), which corresponds to an outflowing velocity of $v_{\text {out }}=0.11 \pm 0.01 c$. The ionization parameter of $\log \xi=4.0_{-0.1}^{+0.7} \mathrm{erg} \mathrm{s}^{-1} \mathrm{~cm}$ indicates that the

$81 \mathrm{Ry} \equiv \frac{m_{e} e^{4}}{8 \varepsilon_{0}^{2} h^{2}}=13.6 \mathrm{eV}$. 


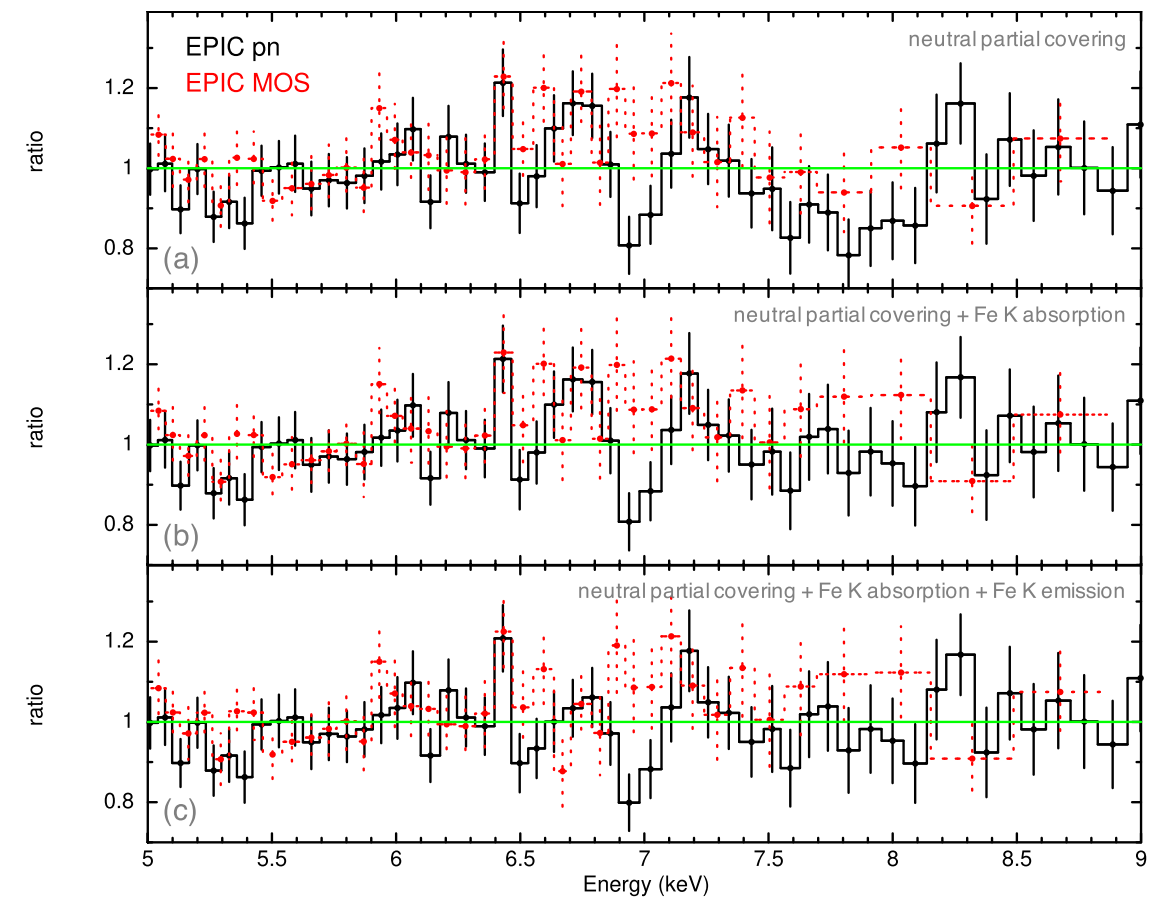

Figure 3. Data-to-model ratios for iron-K models $(E=2-10 \mathrm{keV})$; EPIC pn is plotted in black, the MOS in red. (a) a neutral partial covering absorber model; (b) an Gaussian absorption feature added at $E=7.8 \mathrm{keV}$, which could be indicative of an ultrafast outflow (UFO) due to Fe XXV He $\alpha$ or Fe XXVI Ly $\alpha$; (c) a Gaussian emission feature added at $E=6.7 \mathrm{keV}$. Potential features at $E=6.4,6.95$, and $7.2 \mathrm{keV}$ are not statistically significant.

Table 3

Parameters for the Best-fit Model for $2-10 \mathrm{keV}$

\begin{tabular}{lccccc}
\hline \hline Component & Parameter & Unit & Model & $\chi^{2} / \nu$ & $\Delta \chi^{2} / \Delta \nu$ \\
\hline zpowerlw & $\Gamma$ & & $1.91 \pm 0.02$ & $4255 / 772$ & $\cdots$ \\
& $z^{\mathrm{a}}$ & & 0.0426 & & \\
\hline zpcfabs $^{\mathrm{b}}$ & $N_{\mathrm{H}}$ & $10^{22}$ & $7.03_{-0.29}^{+0.12}$ & $752 / 770$ & $3503 / 2$ \\
& & $\mathrm{~cm}^{-2}$ & & & \\
& Covering & & $0.984_{-0.011}^{+0.014}$ & & \\
& Fraction & & & & \\
& $z^{\mathrm{a}}$ & & 0.0426 & & $13 / 3$ \\
& Line $E$ & $\mathrm{keV}$ & $7.81 \pm 0.12$ & $739 / 767$ & \\
\hline zgauss & $\sigma$ & $\mathrm{keV}$ & $<0.26$ & & \\
& $z^{\mathrm{a}}$ & & 0.0426 & & \\
& $\mathrm{EW}$ & $\mathrm{eV}$ & $-61_{-40}^{+38}$ & & \\
& Line $E$ & $\mathrm{keV}$ & $6.70 \pm 0.06$ & $730 / 765$ & $9 / 2$ \\
zgauss & $\sigma^{\mathrm{a}}$ & $\mathrm{keV}$ & 0.01 & & \\
& $z^{\mathrm{a}}$ & & 0.0426 & & \\
& $\mathrm{EW}$ & $\mathrm{eV}$ & $31 \pm 18$ & & \\
& & & & & \\
\hline
\end{tabular}

Notes. All errors are given at the $90 \%$ level while limits are given at $90 \%$.

${ }^{\text {a }}$ Parameters frozen at their stated values.

$\mathrm{b}$ The covering fraction for the neutral partial covering absorber is purely phenomenological; see Section 4.1 for information about a continuum scattering model.

absorption feature is due to a mixture of both Fe XXV and Fe XXVI (Kallman et al. 2004). The covering fraction of the neutral partial covering absorber remains at $0.984_{-0.010}^{+0.013}$ with full covering excluded at the $90 \%$ level.

\subsection{Relativistic Reflection Model}

Using previous XMM-Newton and NuSTAR observations, $\mathrm{Xu}$ et al. (2017) found evidence for relativistic reflection. Although the lack of a clear broad Fe $\mathrm{K}$ emission line does not support interpreting the spectrum as dominated by relativistic reflection, we test that possibility in order to compare to the results presented in Xu et al. (2017) by using the lamp-post geometry in the relxill code (Dauser et al. 2014; García et al. 2014). This model ( $r e l \times i l l l p)$ considers a lamp-post geometry in which the compact X-ray emitting source is located on the rotation axis of the black hole at a certain height specified in units of gravitational radii, $R_{\mathrm{g}}=G M_{\mathrm{BH}} / c^{2}$. The parameters of this model include: (1) $h$, the height of the source in $R_{\mathrm{g}}$, (2) $a$, the dimensionless spin of the black hole, (3) $i$, the inclination with respect to the normal to the accretion disk, (4) $R_{\text {in }}$, the inner radius of the accretion disk, (5) $R_{\text {out }}$, the outer radius of the accretion disk, (6) $z$, the redshift of the system, (7) $\Gamma$, the power-law index, (8) $\log \xi$, the ionization parameter of the accretion disk, (9) $A_{\mathrm{Fe}}$, the iron abundance of the accretion disk, (10) $E_{\text {cut }}$, the observed high-energy cutoff of the primary spectrum, (11) the reflection fraction (refl_frac), and (12) a model switch controlling the reflection fraction calculation (fixReflFrac).

We assume that the inner radius of the accretion disk extends to the innermost stable circular orbit (ISCO) and a typical outer disk radius of $R_{\text {out }}=400 R_{\mathrm{g}}$. During our analysis, models were not sensitive to the iron abundance, therefore, we fix the iron abundance at solar. We assume an energy cutoff $E_{\text {cut }}=$ $55 \mathrm{keV}$, the value reported by $\mathrm{Xu}$ et al. (2017) whose spectral analysis extended to $E=30 \mathrm{keV}$. We set both the reflection fraction and the fixReflFrac switch to 1 . Throughout our analysis, $\chi^{2}$ was minimized by fixing the height of the illuminating source to $h=2 R_{\mathrm{g}}$, the minimum value permitted by the model.

A model including a neutral partial covering absorber and relxilllp provides a fit of $\chi^{2} / \nu=741.5 / 767$. This is not statistically preferred over the models presented in 
Table 4

Parameters for the Best-fitting XSTAR Model

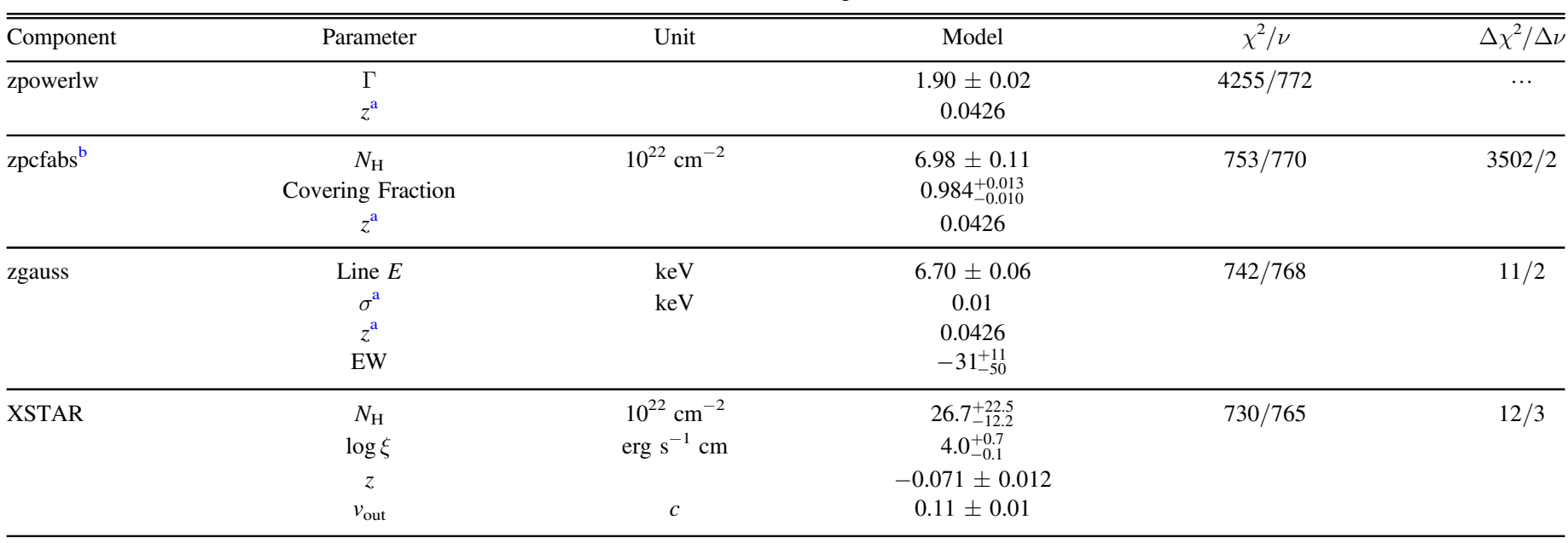

Notes. All errors are given at the $90 \%$ level while limits are given at $90 \%$.

${ }^{a}$ Parameters frozen at their stated values.

$\mathrm{b}$ The covering fraction for the neutral partial covering absorber is purely phenomenological; see Section 4.1 for information about a continuum scattering model.

Sections 4.2 and 4.3 and is, in fact, worse than our model with an absorbed power law and iron-K emission. We still provide full details of this best fit in Table 5 .

\section{NuStar Spectral Analysis}

We perform our spectral analysis using XSPEC v12.10 c (Arnaud 1996) using $\chi^{2}$ statistics. All models take into account Galactic absorption with the tbabs model (Wilms et al. 2000) using a Galactic column density of $N_{\mathrm{H}, \mathrm{Gal}}=1.66 \times 10^{20} \mathrm{~cm}^{-2}$ (Kalberla et al. 2005). All parameters are given in the rest frame of IRAS F05189-2524 $(z=0.0426)$. All errors and limits are given at the level of $90 \%\left(\Delta \chi^{2}=2.7\right.$ for one degree of freedom). Statistical calculations were performed using XSPEC error and steppar commands avoiding local minima when searching $\chi^{2}$ space.

The NUSTAR observation may provide a useful comparison for the results based on the XMM-Newton observation. However, we note that while NUSTAR may place helpful constraints on the high-energy continuum shape and broad spectral features, the energy resolution of $N u S T A R$ is not well suited for the investigation of faint and narrow spectral lines like those found in the $\mathrm{Fe} \mathrm{K}$ region of the XMM-Newton spectra. There is no unusually large flux variability over the course of the full NuSTAR observation; however, it is important to keep in mind the likely variable nature of UFO absorption features (Matzeu et al. 2016). Features observed with XMMNewton may or may not be present (or present with the same strength) during the NuSTAR exposure that is before and after the XMM-Newton observation. The spectra remain signaldominated until $E=20 \mathrm{keV}$, but since our goal is to compare with $X M M-N e w t o n$, we perform our spectral analyses in the mutual energy band from $E=3-10 \mathrm{keV}$ (rest frame). Figure 6 shows the spectrum and background for the full NuSTAR spectrum.

Due to the short exposure time of the NuSTAR observation, which is concurrent with XMM-Newton, the signal-to-noise is not sufficient to detect spectral lines. We therefore focus our analysis on the full NUSTAR spectrum. We begin our examination of the full $N U S T A R$ spectrum by fitting the data with a power-law continuum and neutral partial covering absorber. Figure 7 shows the ratio of this fit along with the $X M M-N e w t o n$ observation. We freeze the covering fraction of the neutral partial absorber to 0.984 . This corresponds to the best-fit value in models of the XMM-Newton observation (see Section 4) where the higher sensitivity in the soft energy band (i.e., $E<3 \mathrm{keV}$ ) provides tighter constraints on the covering fraction. Next, we add the two Gaussian features detected in $X M M-N e w t o n$. Both the central energy values $(E=7.8$ and $6.70 \mathrm{keV})$ and the widths $(\sigma=0.12$ and $0.01 \mathrm{keV})$ of the Gaussian features were frozen to the values found in $X M M$ Newton because they could not be constrained with NuSTAR. We do, however, allow the normalization of each Gaussian feature to vary between $[-1,1] \mathrm{keV}$, thus allowing each Gaussian to be either an emission or absorption feature.

This model provides a reasonable fit to the data with a $\Delta \chi_{\text {red }}^{2}=1.03$. We find a steeper photon index of $\Gamma=2.13 \pm$ 0.09 . The data are consistent with either an emission or absorption feature at the energy of $E=7.81 \mathrm{keV}$, with an equivalent width of $17.1 \mathrm{eV}_{-40.7}^{+37.0} \mathrm{eV}$. Note that at the $90 \%$ level, this is consistent with the XMM-Newton detection of an absorption feature, but the feature is not constrained in NuSTAR alone. The NUSTAR spectrum suggests an emission feature at $E=6.70 \mathrm{keV}$ with an equivalent width of $75 \mathrm{eV} \pm 30 \mathrm{eV}$, also consistent with XMM-Newton at the $90 \%$ level.

\section{Discussion}

\subsection{Accretion Disk Wind}

In Section 4, we report the analysis of the spectrum of IRAS F05189-2524 with a new higher signal-to-noise XMM-Newton observation. We find that modeling the $\mathrm{Fe} \mathrm{K}$ region of the spectrum with a self-consistent photoionization table generated with XSTAR indicates the presence of an outflowing accretion disk wind with a velocity of $v_{\text {out }}=0.11 \pm 0.01 c$.

We can estimate the energetics of the wind following the approach described in Tombesi et al. (2013, 2015, 2017). In our study of the energetics, we will use our best-fit model presented in Section 4.3, comprised of a neutral partial covering absorber, Gaussian Fe $\mathrm{K}$ emission line at $E=6.7 \mathrm{keV}$, and an XSTAR 


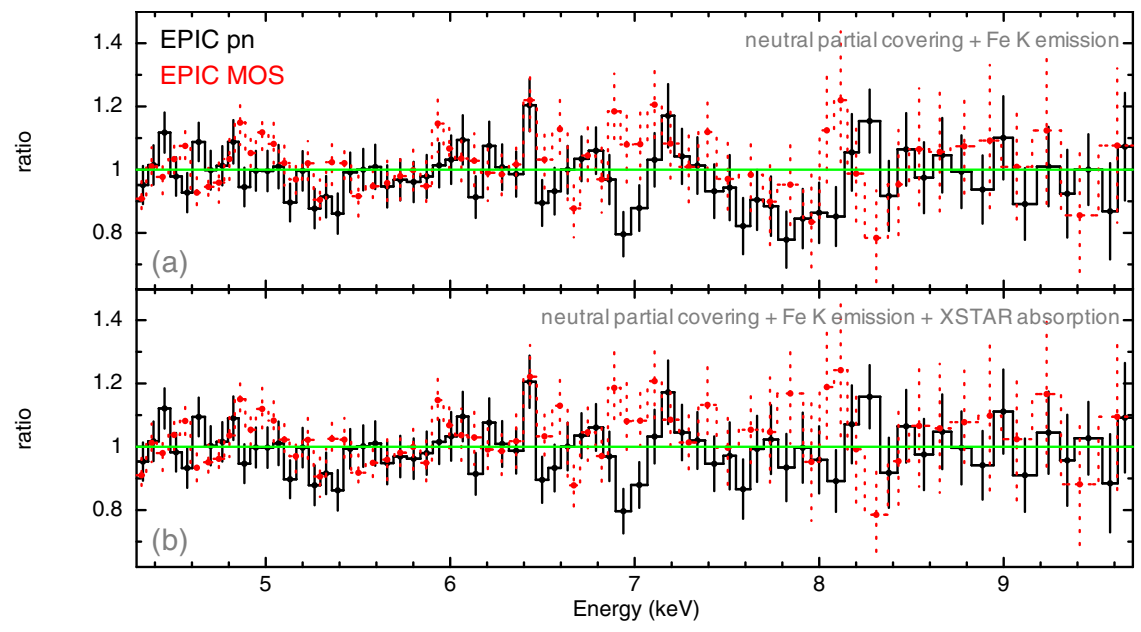

Figure 4. Model residuals are presented for two models that both include neutral partial covering and a Gaussian emission line at $E=6.7 \mathrm{keV}$. The model in the bottom panel includes an XSTAR absorption table which models the absorption feature at $E=7.8 \mathrm{keV}$.

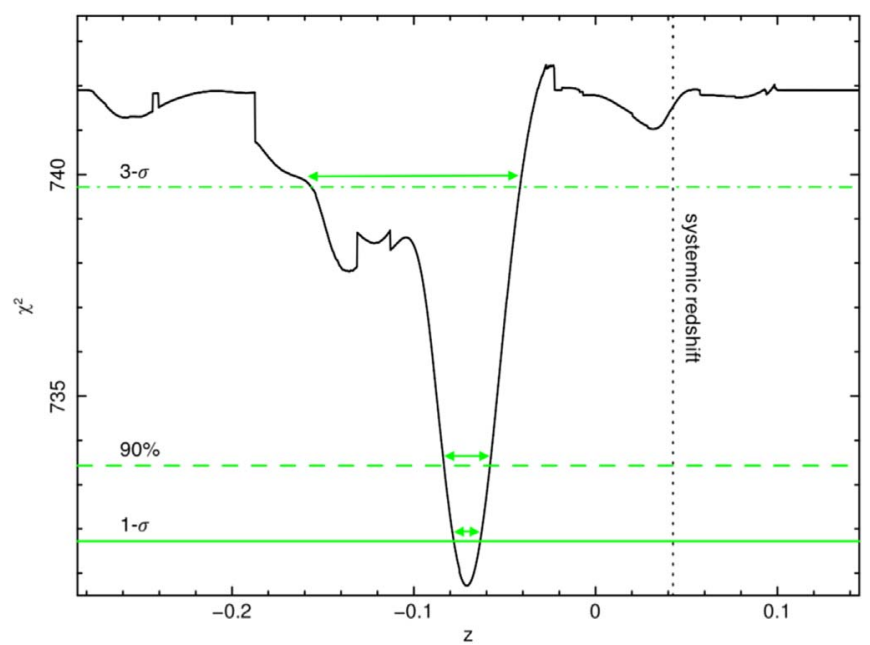

Figure 5. Using the XSPEC steppar command, the $\chi^{2}$ statistic is plotted against the redshift of the XSTAR absorber modeling the Fe $\mathrm{K}$ absorption feature at $7.8 \mathrm{keV}$. The systemic redshift of IRAS F05189-2524 $(z=0.0426)$ is shown with the vertical dotted line. The solid and dashed horizontal lines indicate the $1 \sigma, 90 \%$, and $3 \sigma$ confidence ranges for the value of the redshift of the absorber, which is well constrained at $z=-0.07$ in the observed frame corresponding to an outflowing velocity of $0.11 \mathrm{c}$.

component modeling the $\mathrm{Fe} \mathrm{K}$ absorption feature at $E=$ $7.8 \mathrm{keV}$.

There are multiple published values for the mass of the central SMBH in IRAS F05189-2524. ${ }^{9}$ The photometrically derived black hole mass is estimated to be $M_{\mathrm{BH}}=20.8 \times 10^{7} M_{\odot}$ (Veilleux et al. 2009a). Using the central velocity dispersions measured from the Ca II triplet line widths (Rothberg et al. 2013) and the $M_{\mathrm{BH}^{-}}-\sigma$ relation (Tremaine et al. 2002), the mass is estimated to be $M_{\mathrm{BH}}=42 \times 10^{7} M_{\odot}$ (Xu et al. 2017). Hereafter, we assume the black hole mass calculated in Veilleux et al. (2009a) $M_{\mathrm{BH}}=20.8 \times 10^{7} M_{\odot}$ as a conservative estimate of the black hole mass and thus the X-ray wind energetics.

\footnotetext{
9 Dasyra et al. (2006) derive a dynamical mass estimate of $M_{\mathrm{BH}}=2.95 \times 10^{7}$ $M_{\odot}$ using $\mathrm{CO}$ as a tracer of young stellar velocity dispersions. This method is now understood to systematically underestimate the black hole masses of actively starforming galaxies like IRAS F05189-2524 because the CO is tracing only the young stellar population rather than the older stellar population, whose movement is more indicative of the central mass.
}

A lower limit on the radius of the wind can be derived from the radius at which the observed velocity corresponds to the escape velocity, $r_{\min }=2 G M_{\mathrm{BH}} / v_{\text {out }}^{2} \simeq 5.08 \times 10^{15} \mathrm{~cm}$. Converting to units of Schwarzchild radii $\left(R_{\mathrm{S}}=2 G M_{\mathrm{BH}} / c^{2}\right)$, we obtain a wind launching radius $r \geqslant 83 R_{\mathrm{S}}$ from the central $\mathrm{SMBH}$. An upper limit on the radius of the wind can be derived from the definition of the ionization parameter $(\xi)$ as long as the thickness of the absorber does not exceed its distance to the $\mathrm{SMBH}, N_{\mathrm{H}} \simeq n \Delta r<n r$ (e.g., Crenshaw \& Kraemer 2012). This assumption is consistent with a disk wind observed close to its launching region. Using the XSPEC lumin command and an unabsorbed power-law model, we calculate the ionizing luminosity between 1 and 1000 Ry to be $L_{\text {ion }}=8.15 \times 10^{43} \mathrm{erg}$ $\mathrm{s}^{-1}$. Using the column density and ionization parameter from our best-fit model (Table 4) and the definition of the ionization parameter, we find $r_{\max }=\mathrm{L}_{\mathrm{ion}} / \xi N_{\mathrm{H}}=3.05 \times 10^{16} \mathrm{~cm}$ or $r \leqslant 497 R_{\mathrm{S}}$.

In calculating the energetics, we will only consider the lower limit on the radius of the UFO. Although the estimate of the upper limit is robust, it is far greater than the true location of the outflow. The mass outflow rate of the wind can be estimated considering the equation $\dot{M}_{\text {out }}=4 \pi \mu m_{\mathrm{p}} r N_{\mathrm{H}} C_{\mathrm{F}} v_{\text {out }}$ where $\mu=1.4$ is the mean atomic mass per proton, $m_{\mathrm{p}}$ is the proton mass, and $C_{\mathrm{F}}$ is the wind covering fraction (Crenshaw \& Kraemer 2012). Assuming spherical symmetry, the solid angle subtended by the wind is $\Omega=4 \pi C_{\mathrm{F}}$. We conservatively assume $C_{\mathrm{F}} \simeq 0.5$ estimated from the fraction of sources with detected UFOs and warm absorbers (e.g., Tombesi et al. 2010, 2013; Crenshaw \& Kraemer 2012; Gofford et al. 2013; Tombesi \& Cappi 2014). Using the range of launching radii calculated above, we find a mass outflow rate of $\dot{M}_{\text {out }} \gtrsim$ $1.0 M_{\odot} \mathrm{yr}^{-1}$.

Conservatively assuming that the outflow has reached a terminal velocity, the kinetic (or mechanical) power of the wind can be derived as $\dot{E}_{\mathrm{K}}=\frac{1}{2} \dot{M}_{\text {out }} v_{\text {out }}^{2} \gtrsim 3.6 \times 10^{44} \mathrm{erg} \mathrm{s}^{-1}$. The momentum rate (or force) of the wind is estimated to be $\dot{P}_{\text {out }}=\dot{M}_{\text {out }} v_{\text {out }} \gtrsim 2.2 \times 10^{35}$ dyne. IRAS F05189-2524 has a bolometric luminosity $L_{\mathrm{bol}}=6.47 \times 10^{45} \mathrm{erg} \mathrm{s}^{-1}$ of which $71 \%$ is attributed to the $\mathrm{AGN}\left(L_{\mathrm{AGN}}=4.6 \times 10^{45} \mathrm{erg} \mathrm{s}^{-1}\right.$; Veilleux et al. 2009a). Comparing the wind energetics to the AGN luminosity, we find $\dot{E}_{\mathrm{K}} \gtrsim 8 \% L_{\mathrm{AGN}}$ and $\dot{P}_{\text {out }} \gtrsim 1.4$ $L_{\mathrm{AGN}} / c$. These calculated values are in line with those found in studies with larger samples of disk winds in Seyferts and 
Table 5

Parameters for the Best-fitting relxilllp Model

\begin{tabular}{|c|c|c|c|c|c|}
\hline Component & Parameter & Unit & Model & $\chi^{2} / \nu$ & $\Delta \chi^{2} / \Delta \nu$ \\
\hline zpcfabs $^{a}$ & $\begin{array}{c}N_{\mathrm{H}} \\
\text { Covering Fraction } \\
z^{\mathrm{b}}\end{array}$ & $10^{22} \mathrm{~cm}^{-2}$ & $\begin{array}{c}7.29 \pm 0.12 \\
0.984_{-0.009}^{+0.013} \\
0.0426\end{array}$ & $753 / 770$ & $\cdots$ \\
\hline relxilllp & $\begin{array}{c}h^{\mathrm{b}} \\
a \\
i \\
R_{\text {in }}{ }^{\mathrm{b}} \\
R_{\text {out }} \\
z^{\mathrm{b}} \\
\Gamma \\
\log \xi \\
A_{\mathrm{Fe}}{ }^{\mathrm{b}} \\
E_{\text {cut }} \\
\text { Reflection Fraction }{ }^{\mathrm{b}} \\
\text { Fix Reflection Fraction }\end{array}$ & $\begin{array}{c}R_{\mathrm{g}} \\
\text { degrees } \\
R_{\mathrm{g}} \\
R_{\mathrm{g}} \\
\\
\mathrm{erg} \mathrm{s}^{-1} \mathrm{~cm} \\
\mathrm{solar} \\
\mathrm{keV}\end{array}$ & $\begin{array}{c}<16 \\
0.62_{-0.25}^{+0.13} \\
49 \pm 4 \\
-1 \\
400 \\
0.0426 \\
1.94 \pm 0.03 \\
2.3 \pm 0.5 \\
1 \\
55 \\
1 \\
1\end{array}$ & $742 / 767$ & $\ldots$ \\
\hline
\end{tabular}

Notes. All errors are given at the $90 \%$ level while limits are given at $90 \%$. A full description of model parameters is given in Section 4.4 .

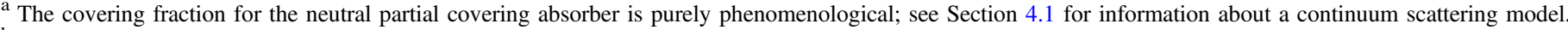

b Parameters frozen at their stated values.

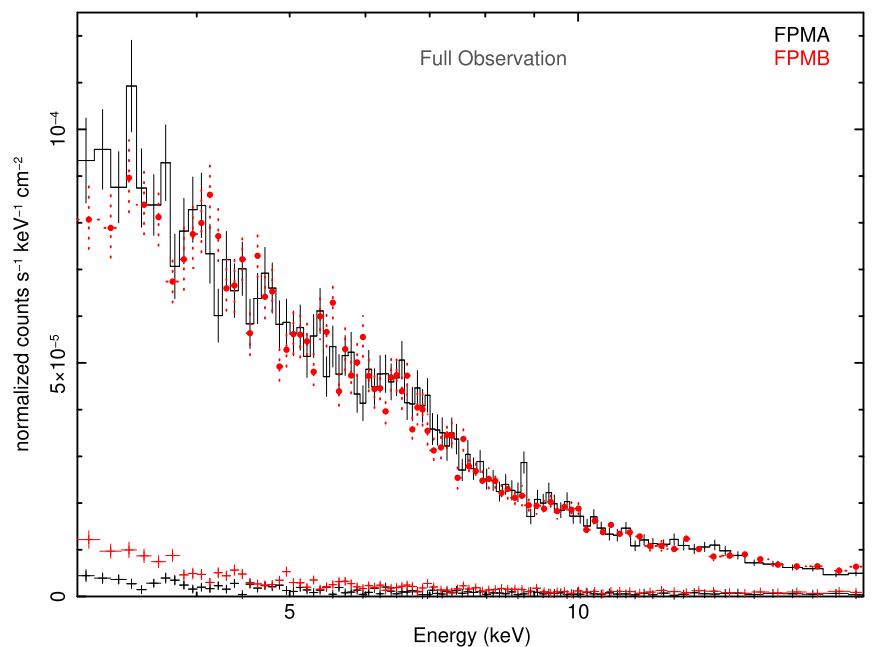

Figure 6. Spectra of the full NuSTAR observation between $E=3-20 \mathrm{keV}$ (rest frame) with the FPMA and B shown in black and red, respectively. The background for each detector is also included.

luminous quasars (e.g., Tombesi et al. 2012, 2015; Gofford et al. 2015; Nardini et al. 2015; Fiore et al. 2017). The accretion disk wind is consistent with having a momentum rate comparable to the AGN radiation pressure, and the energetics are high enough to influence AGN feedback (e.g., Di Matteo et al. 2005; Hopkins \& Elvis 2010; Gaspari et al. 2011).

\subsection{Connection with Galaxy-scale Outflows}

Galaxy-scale outflows have been observed in IRAS F051892524 in neutral, warm ionized, and molecular gas phases (González-Alfonso et al. 2017; Rupke et al. 2017; Fluetsch et al. 2019). Results from the relevant observations are included in Table 6. Energetics derived from the neutral and warm ionized outflowing gas are based on the ground-based integral field spectroscopy (IFS) of Rupke et al. (2017). These observations are limited by the seeing $\left(\sim 1^{\prime \prime}\right)$, which sets an artificial minimum radius $r \sim 400 \mathrm{pc}$. The adopted radius for

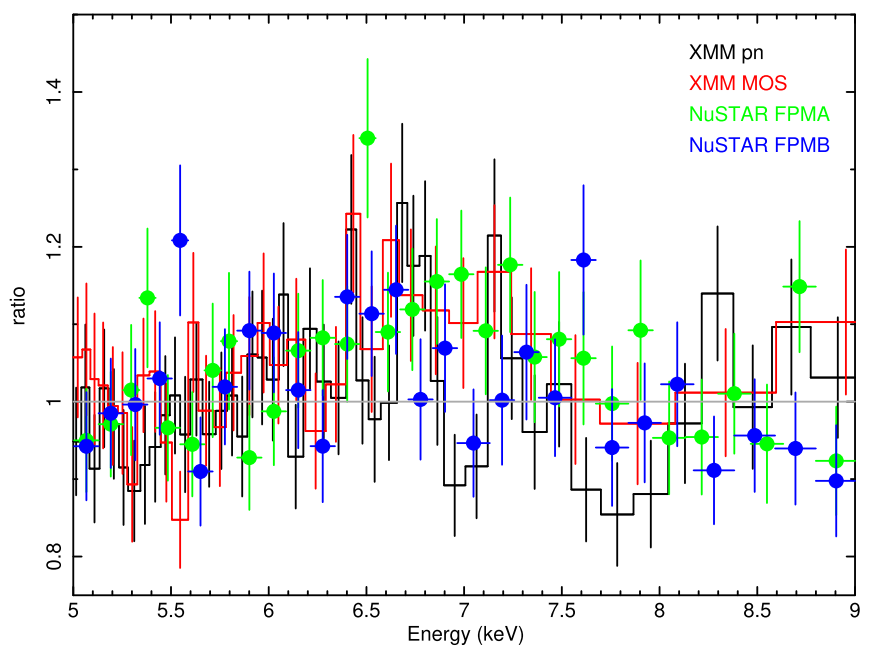

Figure 7. Model ratio of the full NUSTAR and XMM-Newton observations fit with a power law and neutral partial covering absorber. Additional binning applied for visual purposes.

the neutral and warm ionized gas are directly measured from IFS data and are virtually the same. However, a detailed inspection of the neutral and warm ionized gas phases reveals that they differ in spatial distribution. Note that the warm ionized gas phase is negligible compared to the other phases of the large-scale outflow, so it will not be considered any further in our discussion.

The energetics for the molecular outflows are derived using $\mathrm{OH}$ and $\mathrm{CO}$ as tracers for $\mathrm{H}_{2}$. $\mathrm{OH}$ absorption features are detected against the unresolved continuum emission in Herschel far-infrared spectra (González-Alfonso et al. 2017). The dimensions and energetics of the $\mathrm{OH}$ outflow are derived by carefully comparing the velocity profiles of four groundstate and radiatively excited transitions of $\mathrm{OH}$ and the predictions from spherically symmetric radiative transfer models. $\mathrm{OH}$ molecular tracers are sensitive to the dense molecular gas in the nucleus, so this gas component does not extend much beyond $r \sim 500 \mathrm{pc}$. The $\mathrm{CO}$ energetics are 
Table 6

Location and Energetics of The Hot Ionized Disk Wind (UFO) and Multi-phase Galaxy-scale Outflows

\begin{tabular}{|c|c|c|c|c|c|c|}
\hline Gas Phase & $\begin{array}{c}r_{\text {wind }}{ }^{a} \\
(\mathrm{pc})\end{array}$ & $\begin{array}{c}v_{\text {wind }} \mathrm{b}^{\mathrm{b}} \\
\left(\mathrm{km} \mathrm{s}^{-1}\right)\end{array}$ & $\begin{array}{c}\dot{M}_{\text {wind }} \\
\left(M_{\odot} \mathrm{yr}^{-1}\right)\end{array}$ & $\begin{array}{c}\dot{P}_{\text {wind }} \\
\left(10^{34} \text { dyn }\right)\end{array}$ & $\begin{array}{c}\dot{E}_{\mathrm{K}, \text { wind }} \\
\left(10^{42} \mathrm{erg} \mathrm{s}^{-1}\right)\end{array}$ & Reference \\
\hline hot ionized & $0.002-0.010$ & $33,000 \pm 3000$ & $1.0-6.3$ & $22-130$ & $360-2150$ & 1 \\
\hline neutral & 3000 & 560 & $96_{-6}^{+12}$ & $59_{-4}^{+9}$ & $38_{-3}^{+7}$ & 4 \\
\hline warm ionized & 3000 & 423 & $2.5_{-0.69}^{+0.44}$ & $0.78_{-0.22}^{+0.14}$ & $0.21_{-0.05}^{+0.04}$ & 4 \\
\hline $\begin{array}{l}\text { molecular }(\mathrm{CO}) \\
\text { molecular }(\mathrm{OH})\end{array}$ & 189 & 491 & 219 & 68 & 17 & 2 \\
\hline low-velocity & 170 & 200 & 120 & 16 & 1.6 & 3 \\
\hline total & & & $269_{-131}^{+19}$ & $68_{-30}^{+14}$ & $16_{-7}^{+4}$ & 3 \\
\hline
\end{tabular}

Notes. Errors are reported when provided in the appropriate references.

${ }^{a}$ Radius of wind used for calculation of energetics.

${ }^{\mathrm{b}}$ Velocities from Reference 1 are the average over all spaxels of the second component central velocity.

References. (1) This paper, (2) Fluetsch et al. (2019), (3) González-Alfonso et al. (2017), (4) Rupke et al. (2017).

derived from millimeter wave interferometry of spatially resolved $\mathrm{CO}$ emission lines, and the adopted radius is directly measured from these data (Fluetsch et al. 2019). As seen in Table 6, there is good agreement between the different tracers. To simplify our discussion of the energetics, we take the average of the momentum rates for the neutral and two molecular outflows $\left(\dot{P}_{\text {out }, \text { av }}=65 \times 10^{34}\right.$ dyn $)$, as these phases likely provide measurements of the same outflow at different epochs (i.e., distances from the center). We similarly take the average of the outflow velocity for the neutral, molecular $\mathrm{CO}$, and high-velocity molecular OH outflows $\left(v_{\text {out,av }}=534 \mathrm{~km} \mathrm{~s}^{-1}\right)$.

To compare the energetics of the X-ray outflow with the galaxy-scale outflow, we consider two different ways to drive a galaxy-scale outflow. In the case of a momentum-driven outflow, we expect $\dot{P}_{\text {outer }} \simeq \dot{P}_{\text {inner }}$ where "outer" refers to the galaxy-scale outflow and "inner" refers to the inner X-ray wind (Faucher-Giguère \& Quataert 2012; Zubovas \& King 2012). In Section 6.1 , we derive $\dot{P}_{\text {inner }} \gtrsim 22 \times 10^{34}$ dyn, while the momentum rate for the galactic-scale outflows are consistently measured as $\dot{P}_{\text {outer }} \sim 65 \times 10^{34}$ dyn (Table 6). This gives $\dot{P}_{\text {outer }} / \dot{P}_{\text {inner }} \sim 3$; however, given the large uncertainties in the momentum rate estimates, our data are not inconsistent with a momentum-driving scenario.

For an energy-driven outflow, conservation of energy gives $\frac{1}{2} \dot{M}_{\text {inner }} v_{\text {inner }}^{2}=\frac{1}{2} f \dot{M}_{\text {outer }} v_{\text {outer }}^{2}$ where "outer" refers to the galaxyscale outflow and "inner" refers to the inner X-ray wind. The efficiency factor, $f$, is limited to $[0,1]$ where $f=0$ and $f=1$ are two extremes indicating either full dissipation or conservation of kinetic power within the outflow, respectively. Using the expression for the momentum rate, this can be rewritten as $\dot{P}_{\text {inner }} v_{\text {inner }}=f \dot{P}_{\text {outer }} v_{\text {outer }}$. Thus, the expected momentum rate for the large-scale outflow in an energy-driven outflow is given as $\dot{P}_{\text {outer }}=f\left(v_{\text {inner }} / v_{\text {outer }}\right) \dot{P}_{\text {inner }}$. The efficiency factor can be interpreted as the ratio between the covering fractions of the inner and outer outflows or the fraction of the kinetic energy of the inner X-ray wind that goes into bulk motion of the swept-up molecular material.

Using average values for the large-scale galactic outflows along with the lower limit of the momentum rate for the UFO, we obtain $f=0.05$. This low efficiency value could be the result of a highly clumpy ISM or if the covering fraction of the large-scale outflow is low (Wagner et al. 2012, 2013; Hopkins et al. 2016). We note that the ratio of the momentum rate of the molecular outflow to the momentum rate of UFO

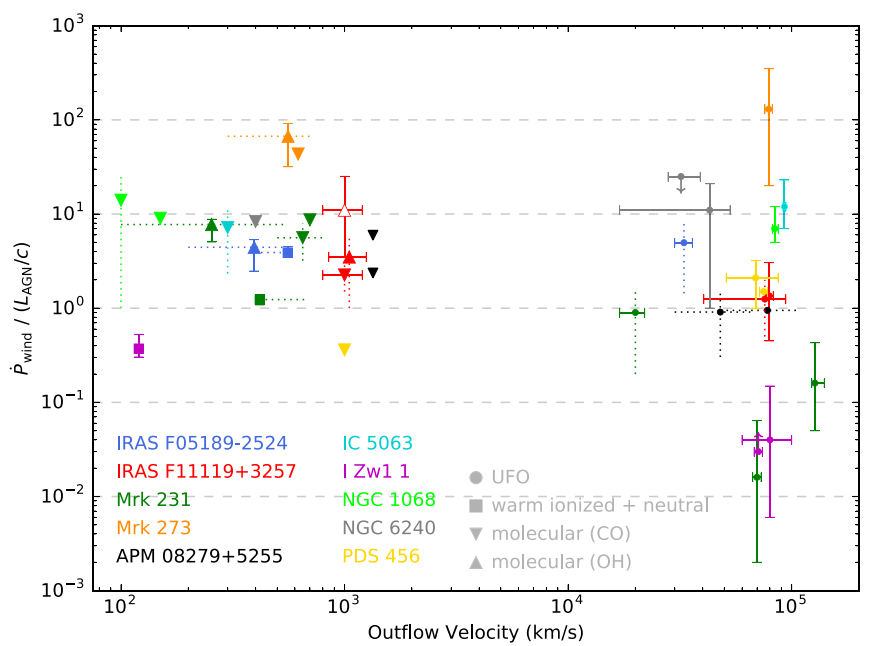

Figure 8. Momentum rate $\left(\dot{P}_{\text {wind }}\right)$ normalized by the momentum of the radiation $\left(L_{\mathrm{AGN}} / c\right)$ is plotted against the wind outflow velocity for 10 objects with observed UFOs and large-scale galactic outflows with good constraints on their spatial scales. Solid error bars indicate that upper and lower errors were calculated, whereas dotted error bars indicate that only a range of values was provided. Arrows indicate limits. UFO measurements are plotted as circles, warm ionized and neutral gas as squares, the molecular (CO) as downward triangles, and the molecular $(\mathrm{OH})$ as upward triangles. For molecular measurements, filled symbols indicate a time-averaged momentum rate, whereas an open symbol is an "instantaneous" or local momentum rate. See Table 7 in the Appendix for more details and references for each specific object.

$\left(\dot{P}_{\mathrm{mol}} / \dot{P}_{\mathrm{UFO}} \sim 0.5-3\right)$ is approximately of order unity within the errors. Richings \& Faucher-Giguère (2018) find that $\dot{P}_{\mathrm{mol}} / \dot{P}_{\mathrm{UFO}}$ of order unity could still be attributed to an energy-driven outflow where the thermalized mechanical energy is mostly lost through efficient cooling due to in situ formation of molecular gas within the outflow.

Additionally, we note that a purely IR radiation driven molecular outflow (as opposed to mechanical acceleration; see e.g., King \& Pounds 2015) is not preferred, but not strictly ruled out. In such a scenario, the momentum of the molecular outflow is given by $\dot{P}_{\mathrm{mol}} \sim\left(1+\eta \tau_{\mathrm{IR}}\right)\left(L_{\mathrm{IR}} / c\right)$ where theoretically $\eta \sim 0.5-0.9$ (Zhang \& Davis 2017; Ishibashi et al. 2018) and $\tau_{\text {IR }}$ is the optical depth in the infrared. For IRAS F05189$2524, L_{\mathrm{IR}}=1.38 \times 10^{12} L_{\odot}$ (González-Alfonso et al. 2017) 
which implies $\tau_{\text {IR }} \sim 3-5$, and thus requires significant IR trapping.

Finally, we consider IRAS F05189-2524 in the context of nine other sources that have observed UFOs and large-scale galactic outflows with good constraints on their spatial scales. Figure 8 shows the momentum rate against the velocity of the outflow for IRAS F05189-2529 and nine other sources. Detailed information and references for each object is included in Table 7 in the Appendix. It is clear that some objects reside in the momentum-driven regime while others are more consistent with the energy-driven scenario, suggesting that there is a range of efficiency factors $(f \sim 0.001-0.5)$ that likely depend on specific physical conditions in each object.

\section{Conclusions}

We present new XMM-Newton and NuSTAR observations of the galaxy merger IRAS F05189-2524, a ULIRG and optical Seyfert 2. Testing multiple spectral models yields a best-fit model consisting of a highly ionized absorber with either an absorbed power law and neutral partial covering absorber or a neutral absorber and scattered emission. We find evidence for a blueshifted $\mathrm{Fe} \mathrm{K}$ absorption feature at $E=7.8 \mathrm{keV}$ (rest frame), which implies a UFO with $v_{\text {out }}=0.11 \pm 0.01 c$.

We calculate that the UFO has a mass outflow rate of $\dot{M}_{\text {out }} \gtrsim$ $1.0 M_{\odot} \mathrm{yr}^{-1}$, a kinetic power of $\dot{E}_{\mathrm{K}} \gtrsim 3.6 \times 10^{44} \mathrm{erg} \mathrm{s}^{-1}(8 \%$ $L_{\mathrm{AGN}}$ ), and a momentum rate (or force) of $\dot{P}_{\text {out }} \gtrsim 22 \times 10^{34}$ dyne $\left(1.4 L_{\mathrm{AGN}} / c\right)$. Observed large-scale galactic outflows in IRAS F05189-2524 have an average momentum rate of $\dot{P}=68 \times 10^{34}$ dyne, yielding $\dot{P}_{\text {inner }} / \dot{P}_{\text {outer }} \sim 3$. Given the large uncertainties in the momentum rate estimates, $\dot{P}_{\text {inner }} / \dot{P}_{\text {outer }}$ is not inconsistent with unity, or a momentum-driven scenario. In the energy-driven outflow scenario, the fraction $f$ of the kinetic energy in the inner X-ray wind that goes into bulk motion of the large-scale outflow is $f \sim 0.05$. Such a low efficiency could be attributed to a highly clumpy ISM or if the covering fraction of the large-scale outflow is low or if the hot gas has efficiently cooled leading to an in situ formation of the molecular outflow.

We compare the outflow in IRAS F05189-2524 to nine other objects with observed UFOs and large-scale galactic outflows with solid constraints on the outflow energetics. We find that there is a range of efficiency factors $(f \sim 0.001-0.5)$ and driving mechanisms that likely depend on specific physical conditions in each object. While this remains a small sample, it is an important step toward building a comprehensive sample that can be used to further probe the complex relationships of AGNs and galaxy co-evolution.

R.N.S. and S.V. acknowledge support from NASA, grant number 15-NUSTAR215-0021. F.T. acknowledges support by the Programma per Giovani Ricercatori-anno 2014 "Rita Levi Montalcini." S.V. acknowledges support from a Raymond and Beverley Sackler Distinguished Visitor Fellowship and thanks the host institute, the Institute of Astronomy, where this work was concluded. S.V. also acknowledges support by the Science and Technology Facilities Council (STFC) and by the Kavli Institute for Cosmology, Cambridge. A.L. acknowledges financial support from the Italian Space Agency (ASI) under the contract ASI-INAF n.2017-14-H.0.

Facility: XMM-Newton (EPIC).

\section{Appendix \\ Outflow Energetics of the AGN Sample}

In Table 7, we provide additional information and references for the 10 objects with observed ultrafast outflows and largescale galactic outflows that are shown in Figure 8. The largescale outflows in all of these objects have good constraints on their spatial scales.

Table 7

Outflow Velocities and Momentum Rates for Objects With Observed Ultrafast Outflows and Large-scale Galactic Outflows

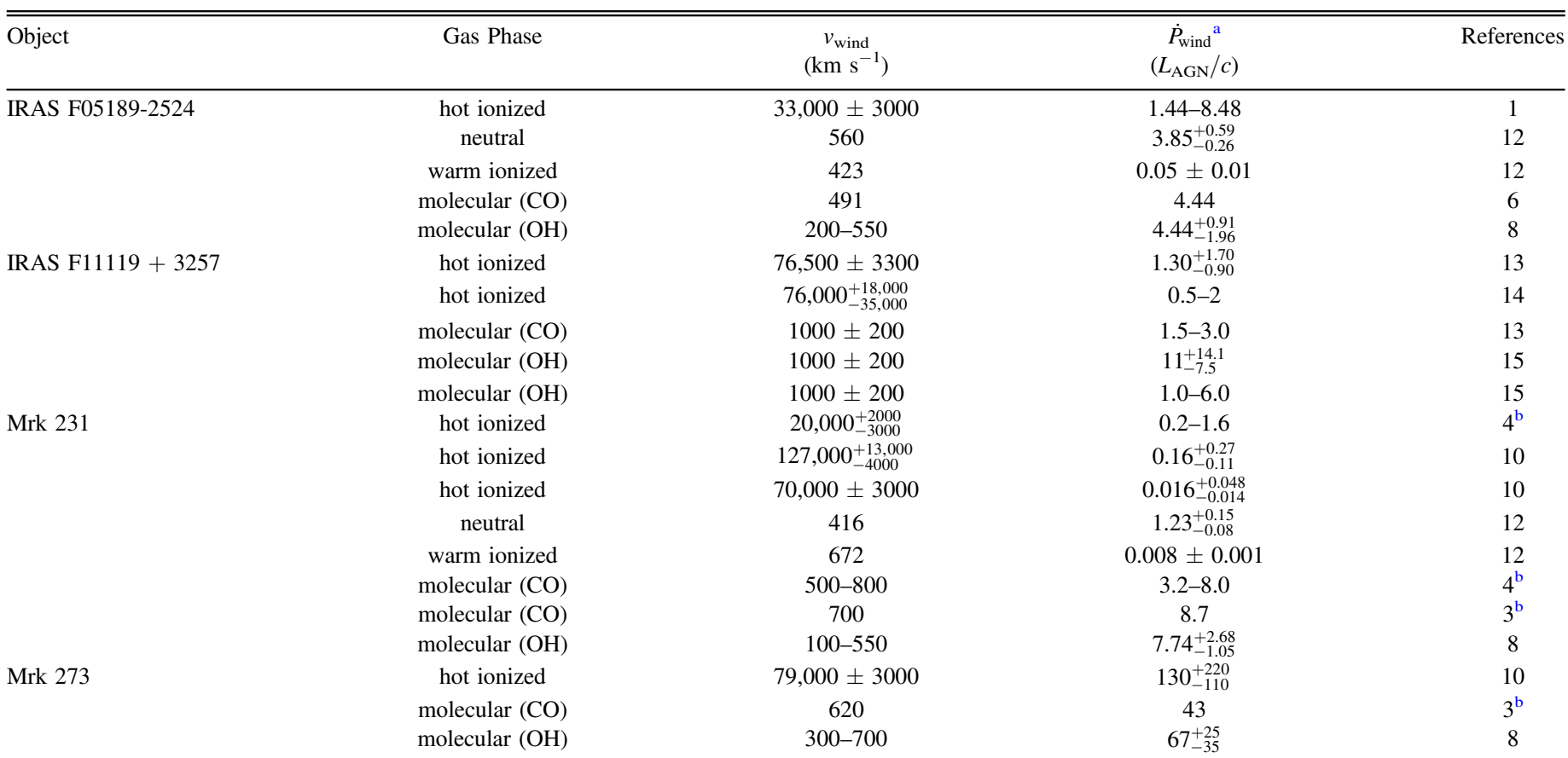


Table 7

(Continued)

\begin{tabular}{|c|c|c|c|c|}
\hline Object & Gas Phase & $\begin{array}{c}v_{\text {wind }} \\
\left(\mathrm{km} \mathrm{s}^{-1}\right)\end{array}$ & $\begin{array}{c}\dot{P}_{\text {wind }}{ }^{\mathrm{a}} \\
\left(L_{\mathrm{AGN}} / c\right)\end{array}$ & References \\
\hline \multirow[t]{4}{*}{ APM $08279+5255$} & hot ionized & $48,000-108,000$ & 0.95 & 5 \\
\hline & hot ionized & $30,000-66,000$ & $0.3-1.5$ & 5 \\
\hline & molecular (CO) & 1340 & 2.37 & 5 \\
\hline & molecular (CO) & 1340 & 5.97 & 5 \\
\hline \multirow[t]{2}{*}{ IC 5063} & hot ionized & $93,000_{-1400}^{+1300}$ & $12_{-5}^{+11}$ & 10 \\
\hline & molecular (CO) & 300 & $2.3-12.0$ & $3^{\mathrm{b}}$ \\
\hline \multirow[t]{3}{*}{ I Zw 1} & hot ionized & $80,000 \pm 20,000$ & $0.04_{-0.03}^{+0.11}$ & 10 \\
\hline & hot ionized & $71,000 \pm 3000$ & $>0.03$ & 10 \\
\hline & neutral & 120 & $0.37_{-0.07}^{+0.15}$ & 12 \\
\hline \multirow[t]{3}{*}{ NGC 1068} & hot ionized & $84,000_{-2000}^{+3000}$ & $7_{-2}^{+5}$ & 10 \\
\hline & molecular (CO) & 100 & $1-27$ & 7 \\
\hline & molecular (CO) & 150 & 9 & $3^{\mathrm{b}}$ \\
\hline \multirow[t]{3}{*}{ NGC 6240} & hot ionized & $43,000_{-26,000}^{+10,000}$ & $11 \pm 10$ & 10 \\
\hline & hot ionized & $32,000_{-4000}^{+7,000}$ & $<25$ & 10 \\
\hline & molecular (CO) & 400 & 8 & $3^{\mathrm{b}}$ \\
\hline \multirow[t]{3}{*}{ PDS 456} & hot ionized & $69,000 \pm 18,000$ & $2.1 \pm 1.1$ & 9 \\
\hline & hot ionized & $75,000 \pm 3000$ & 1.5 & 11 \\
\hline & molecular $(\mathrm{CO})$ & 1000 & 0.36 & 2 \\
\hline
\end{tabular}

Notes. Errors are presented when published by their respective authors. For simplicity, objects with both warm ionized and neutral observed outflows were plotted as a single data point in Figure 8.

${ }^{\text {a }} \dot{P}_{\text {wind }}=\dot{M}_{\text {wind }} v_{\text {wind }} ; \dot{E}_{\text {wind }}=\frac{1}{2} \dot{M}_{\text {wind }} v_{\text {wind }}^{2}$.

${ }^{\mathrm{b}}$ CO-based molecular outflow momentum rates from these references were divided by a factor of 3 so that they are on the same scale as the other measurements. References. (1) This paper, (2) Bischetti et al. (2019), (3) Cicone et al. (2014), (4) Feruglio et al. (2015), (5) Feruglio et al. (2017), (6) Fluetsch et al. (2019), (7) García-Burillo et al. (2014), (8) González-Alfonso et al. (2017), (9) Luminari et al. (2018), (10) Mizumoto et al. (2019), (11) Nardini et al. (2015), (12) Rupke et al. (2017), (13) Tombesi et al. (2015), (14) Tombesi et al. (2017), (15) Veilleux et al. (2017).

\section{ORCID iDs}

Robyn N. Smith (10 https://orcid.org/0000-0001-5626-5209 Francesco Tombesi (iD https://orcid.org/0000-0002-6562-8654 Sylvain Veilleux (i) https://orcid.org/0000-0002-3158-6820

\section{References}

Arnaud, K. A. 1996, adass V, 101, 17

Asplund, M., Grevesse, N., Sauval, A. J., et al. 2009, ARA\&A, 47, 481

Bellocchi, E., Arribas, S., Colina, L., et al. 2013, A\&A, 557, A59

Berti, E., \& Volonteri, M. 2008, ApJ, 684, 822

Bischetti, M., Piconcelli, E., Feruglio, C., et al. 2019, A\&A, 628, A118

Braito, V., Reeves, J. N., Matzeu, G. A., et al. 2018, MNRAS, 479, 3592

Cicone, C., Maiolino, R., Sturm, E., et al. 2014, A\&A, 562, A21

Crenshaw, D. M., \& Kraemer, S. B. 2012, ApJ, 753, 75

Dasyra, K. M., Tacconi, L. J., Davies, R. I., et al. 2006, ApJ, 651, 835

Dauser, T., Garcia, J., Parker, M. L., et al. 2014, MNRAS, 444, L100

Di Matteo, T., Springel, V., \& Hernquist, L. 2005, Natur, 433, 604

Fabian, A. C. 1999, MNRAS, 308, L39

Faucher-Giguère, C.-A., \& Quataert, E. 2012, MNRAS, 425, 605

Ferrarese, L., \& Merritt, D. 2000, ApJL, 539, L9

Feruglio, C., Ferrara, A., Bischetti, M., et al. 2017, A\&A, 608, A30

Feruglio, C., Fiore, F., Carniani, S., et al. 2015, A\&A, 583, A99

Fiore, F., Feruglio, C., Shankar, F., et al. 2017, A\&A, 601, A143

Fluetsch, A., Maiolino, R., Carniani, S., et al. 2019, MNRAS, 483, 4586

Freyberg, M. J., Briel, U. G., Dennerl, K., et al. 2004, Proc. SPIE , 5165, 112

García-Burillo, S., Combes, F., Usero, A., et al. 2014, A\&A, 567, A125

García, J., Dauser, T., Lohfink, A., et al. 2014, ApJ, 782, 76

Gaspari, M., Brighenti, F., D'Ercole, A., et al. 2011, MNRAS, 415, 1549

Gebhardt, K., Bender, R., Bower, G., et al. 2000, ApJL, 539, L13

Gofford, J., Reeves, J. N., McLaughlin, D. E., et al. 2015, MNRAS, 451, 4169

Gofford, J., Reeves, J. N., Tombesi, F., et al. 2013, MNRAS, 430, 60

González-Alfonso, E., Fischer, J., Spoon, H. W. W., et al. 2017, ApJ, 836, 11

Harrison, F. A., Craig, W. W., Christensen, F. E., et al. 2013, ApJ, 770, 103

Hopkins, P. F., \& Elvis, M. 2010, MNRAS, 401, 7
Hopkins, P. F., Hernquist, L., Cox, T. J., et al. 2006, ApJS, 163, 1 Hopkins, P. F., Torrey, P., Faucher-Giguère, C.-A., et al. 2016, MNRAS, 458,816

Ishibashi, W., Fabian, A. C., \& Maiolino, R. 2018, MNRAS, 476, 512

Jansen, F., Lumb, D., Altieri, B., et al. 2001, A\&A, 365, L1

Kalberla, P. M. W., Burton, W. B., Hartmann, D., et al. 2005, A\&A, 440, 775

Kallman, T., \& Bautista, M. 2001, ApJS, 133, 221

Kallman, T. R., Palmeri, P., Bautista, M. A., et al. 2004, ApJS, 155, 675

King, A. 2003, ApJL, 596, L27

King, A., \& Pounds, K. 2015, ARA\&A, 53, 115

King, A. R., \& Pounds, K. A. 2003, MNRAS, 345, 657

Koss, M., Mushotzky, R., Baumgartner, W., et al. 2013, ApJL, 765, L26

Liu, W., Veilleux, S., Iwasawa, K., et al. 2019, ApJ, 872, 39

Longinotti, A. L., Krongold, Y., Guainazzi, M., et al. 2015, ApJL, 813, L39

Luminari, A., Piconcelli, E., Tombesi, F., et al. 2018, A\&A, 619, A149

Matzeu, G. A., Reeves, J. N., Nardini, E., et al. 2016, MNRAS, 458, 1311

Mizumoto, M., Izumi, T., \& Kohno, K. 2019, ApJ, 871, 156

Moderski, R., \& Sikora, M. 1996, MNRAS, 283, 854

Nandra, K., \& Pounds, K. A. 1994, MNRAS, 268, 405

Nardini, E., Reeves, J. N., Gofford, J., et al. 2015, Sci, 347, 860

Nardini, E., Wang, J., Fabbiano, G., et al. 2013, ApJ, 765, 141

Paggi, A., Fabbiano, G., Risaliti, G., et al. 2017, ApJ, 841, 44

Parker, M. L., Pinto, C., Fabian, A. C., et al. 2017, Natur, 543, 83

Ptak, A., Heckman, T., Levenson, N. A., et al. 2003, ApJ, 592, 782

Reeves, J. N., \& Turner, M. J. L. 2000, MNRAS, 316, 234

Richings, A. J., \& Faucher-Giguère, C.-A. 2018, MNRAS, 478, 3100

Risaliti, G., Gilli, R., Maiolino, R., et al. 2000, A\&A, 357, 13

Rothberg, B., Fischer, J., Rodrigues, M., et al. 2013, ApJ, 767, 72

Rupke, D. S., Veilleux, S., \& Sanders, D. B. 2005, ApJ, 632, 751

Rupke, D. S. N., Gültekin, K., \& Veilleux, S. 2017, ApJ, 850, 40

Rupke, D. S. N., \& Veilleux, S. 2013, ApJ, 768, 75

Rupke, D. S. N., \& Veilleux, S. 2015, ApJ, 801, 126

Sanders, D. B., Soifer, B. T., Elias, J. H., et al. 1988, ApJ, 325, 74

Severgnini, P., Risaliti, G., Marconi, A., et al. 2001, A\&A, 368, 44

Silk, J., \& Rees, M. J. 1998, A\&A, 331, L1

Strüder, L., Briel, U., Dennerl, K., et al. 2001, A\&A, 365, L18 
Sturm, E., González-Alfonso, E., Veilleux, S., et al. 2011, ApJL, 733, L16 Tarter, C. B., Tucker, W. H., \& Salpeter, E. E. 1969, ApJ, 156, 943

Teng, S. H., Rigby, J. R., Stern, D., et al. 2015, ApJ, 814, 56

Teng, S. H., Veilleux, S., Anabuki, N., et al. 2009, ApJ, 691, 261

Teng, S. H., Veilleux, S., \& Baker, A. J. 2013, ApJ, 765, 95

Tombesi, F., \& Cappi, M. 2014, MNRAS, 443, L104

Tombesi, F., Cappi, M., Reeves, J. N., et al. 2010, A\&A, 521, A57

Tombesi, F., Cappi, M., Reeves, J. N., et al. 2011, ApJ, 742, 44

Tombesi, F., Cappi, M., Reeves, J. N., et al. 2012, MNRAS, 422, L1

Tombesi, F., Cappi, M., Reeves, J. N., et al. 2013, MNRAS, 430, 1102

Tombesi, F., Meléndez, M., Veilleux, S., et al. 2015, Natur, 519, 436

Tombesi, F., Veilleux, S., Meléndez, M., et al. 2017, ApJ, 850, 151

Tremaine, S., Gebhardt, K., Bender, R., et al. 2002, ApJ, 574, 740

Turner, M. J. L., Abbey, A., Arnaud, M., et al. 2001, A\&A, 365, L27

Veilleux, S., Bolatto, A., Tombesi, F., et al. 2017, ApJ, 843, 18

Veilleux, S., Kim, D.-C., Peng, C. Y., et al. 2006, ApJ, 643, 707
Veilleux, S., Kim, D.-C., Rupke, D. S. N., et al. 2009a, ApJ, 701, 587

Veilleux, S., Kim, D.-C., \& Sanders, D. B. 1999a, ApJ, 522, 113

Veilleux, S., Kim, D.-C., \& Sanders, D. B. 2002, ApJS, 143, 315

Veilleux, S., Meléndez, M., Sturm, E., et al. 2013, ApJ, 776, 27

Veilleux, S., Rupke, D. S. N., Kim, D.-C., et al. 2009b, ApJS, 182, 628

Veilleux, S., Sanders, D. B., \& Kim, D.-C. 1999b, ApJ, 522, 139

Veilleux, S., Teng, S. H., Rupke, D. S. N., et al. 2014, ApJ, 790, 116

Volonteri, M., Madau, P., Quataert, E., et al. 2005, ApJ, 620, 69

Wagner, A. Y., Bicknell, G. V., \& Umemura, M. 2012, ApJ, 757, 136

Wagner, A. Y., Umemura, M., \& Bicknell, G. V. 2013, ApJL, 763, L18

Westmoquette, M. S., Clements, D. L., Bendo, G. J., et al. 2012, MNRAS, 424,416

Wilms, J., Allen, A., \& McCray, R. 2000, ApJ, 542, 914

Xu, Y., Baloković, M., Walton, D. J., et al. 2017, ApJ, 837, 21

Zhang, D., \& Davis, S. W. 2017, ApJ, 839, 54

Zubovas, K., \& King, A. 2012, ApJL, 745, L34 